\title{
CONFECÇÃO DE PILOROS ARTIFICIAIS EM ÍLEO TERMINAL SEM SECÇÃO DE MUSCULATURA EM RATOS: ESTUDO ANÁTOMO-PATOLÓGICO
}

\author{
ARTIFICIAL PYLORUS WITH NO MUSCULATURE SECTION IN TERMINAL \\ BOWEL IN RATS: ANATOMO-PATHOLOGICAL STUDY
}

\author{
Sergio Ibañez Nunes, ACBC-MG ${ }^{1}$ \\ Lucélia Rita Gaudino Caputo \\ Alcino Lázaro da Silva, TCBC-MG ${ }^{3}$
}

\begin{abstract}
RESUMO: Objetivo: Estudar, experimentalmente, a diminuição do trânsito intestinal através de piloros artificiais no íleo terminal de ratos, sem secção da musculatura entérica. Método: O estudo foi realizado em 40 ratos distribuídos em dois grupos de 20 animais cada. Foram confeccionados quatro piloros no íleo terminal de cada animal, com pontos sero-musculares separados, distribuídos circunferencialmente na alça intestinal. O Grupo 1 foi morto com 15 dias e o Grupo 2, com 30 dias. Aferimos as medidas da circunferência do intestino no transoperatório e no momento da necrópsia. Resultados: No Grupo 1 houve dilatação média de $3 \mathrm{~mm}$ no nível do primeiro piloro e de $4,15 \mathrm{~mm}$ no quarto piloro. No Grupo 2 a dilatação média foi de $7,50 \mathrm{~mm}$ no primeiro piloro e de $5,75 \mathrm{~mm}$ no quarto piloro. No estudo anátomo-patológico ficou evidente a formação bem definida dos piloros. Conclusão: Não é necessário remover ou seccionar a musculatura do intestino delgado, nem a secção do plexo nervoso próprio do intestino, para promover a dilatação intestinal com esse método e, como consequência, diminuir o trânsito intestinal.
\end{abstract}

Descritores: Íleo; Piloro; Trânsito gastroenterológico

\section{INTRODUÇÃO}

Em algumas situações não fisiológicas, como na síndrome de "dumping", na síndrome do intestino curto, nas ileostomias e na anastomose íleo-anal, a necessidade de diminuir o trânsito do intestino delgado se faz presente para aumentar a absorção dos nutrientes, bem como de água e eletrólitos ${ }^{1}$.

Diversos autores estudaram métodos com objetivo de diminuir o trânsito intestinal, como os piloros artificiais. A maioria dos pesquisadores enfatiza a retirada ou secção da musculatura, de uma ${ }^{2}$ ou das duas ${ }^{3}$ camadas musculares, bem como a importância da secção dos plexos nervosos próprios do intestino, com a finalidade de inibir ou interromper o movimento peristáltico.

Este estudo tem por objetivo estudar experimentalmente em ratos, piloros artificiais em íleo,sem secção da musculatura ou de serosa no intuito de diminuir o trânsito intestinal.

\footnotetext{
1. Professor de Técnica Operatória e Cirurgia Experimental da UFJF, Mestre em Cirurgia Experimental

2. Professora de Anatomia Patológica da Universidade de Alfenas, Mestre em Anatomia Patológica

3. Professor Titular de Cirurgia do Aparelho Digestivo da UFMG
}

\section{Recebido em14/05/2001}

Aceito para publicação em 13/08/2002

Trabalho realizado no Departamento de Técnica Operatória e Cirurgia Experimental da Universidade Federal de Juiz de Fora e no Departamento de Anatomia Patológica da Universidade de Alfenas 


\section{MÉTODO}

Foram utilizados 40 ratos da raça Wistar, de dois meses de idade, nascidos na mesma data, fêmeas, com peso entre 250 gramas e 350 gramas, do Biotério da Faculdade de Biologia da Universidade Federal de Juiz de Fora.

Os animais foram divididos em dois grupos, assim distribuídos: Grupo 1: 20 ratos abatidos no $15^{\circ}$ dia de pós-operatório; Grupo 2: 20 ratos abatidos no $30^{\circ}$ dia de pós-operatório.

Nos 40 ratos foram confeccionados quatro piloros no íleo terminal de cada animal. Tendo como referência o piloro ileocecal, a cada $5 \mathrm{~cm}$ realizamos um piloro artificial, desta forma, o primeiro piloro distou $20 \mathrm{~cm}$ do piloro ileocecal.

Os piloros foram realizados com pontos separados sero-musculares de fio agulhado de seda trançada número 4-0, com três seminós em cada ponto. Realizamos quatro pontos, dispostos circunfe- rencialmente, para cada piloro. Cada ponto foi confeccionado atingindo as camadas sero-musculares em aproximadamente $2 \mathrm{~mm}$, compreendendo na verdade duas perfurações, distando $4 \mathrm{~mm}$ uma da outra. Este segmento de $4 \mathrm{~mm}$, entre as duas perfurações, foi invaginado para, no conjunto, confeccionar cada piloro.

No transoperatório foram feitas medidas da circunferência da alça do íleo a $1 \mathrm{~mm}$ a montante do primeiro piloro e do quarto piloro, utilizando-se um fio de sutura que foi cortado no tamanho da circunferência e medido em seguida. Novas medidas, nos mesmos locais foram realizadas, na data da morte de cada animal (Figura-1).

Um segmento de alça intestinal compreendendo 10 centímetros antes do $1^{\circ}$ piloro até o ceco, inclusive, foi retirado sem o mesentério, aberto longitudinalmente na face antimesentérica, e lavado em água corrente. Essa alça intestinal aberta foi presa em papelão com alfinetes e fixada em solução de um

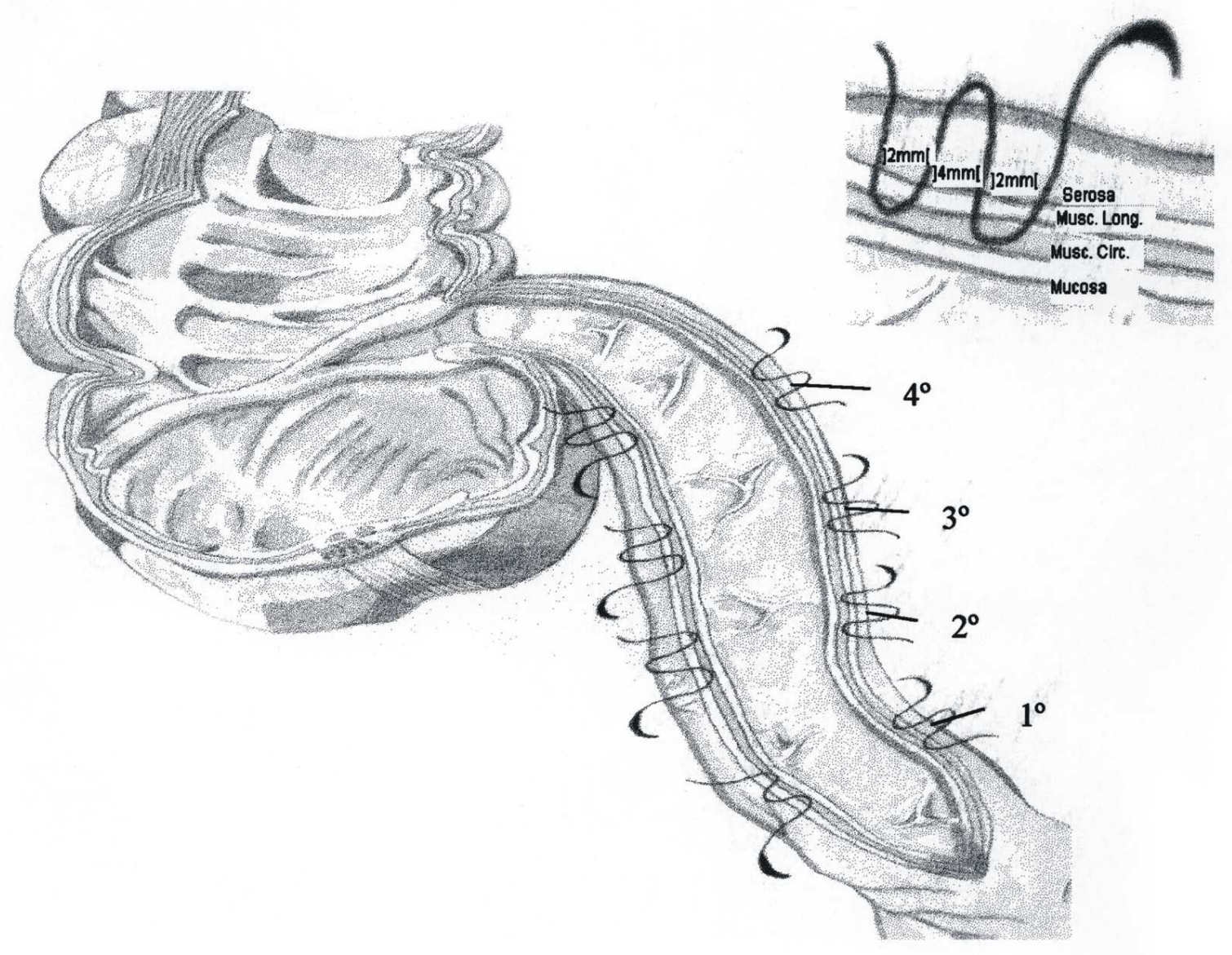

Figura 1 - Fios de sutura passados na parede do intestino sem atingir a mucosa. No detalhe as distâncias entre os pontos seromusculares. 

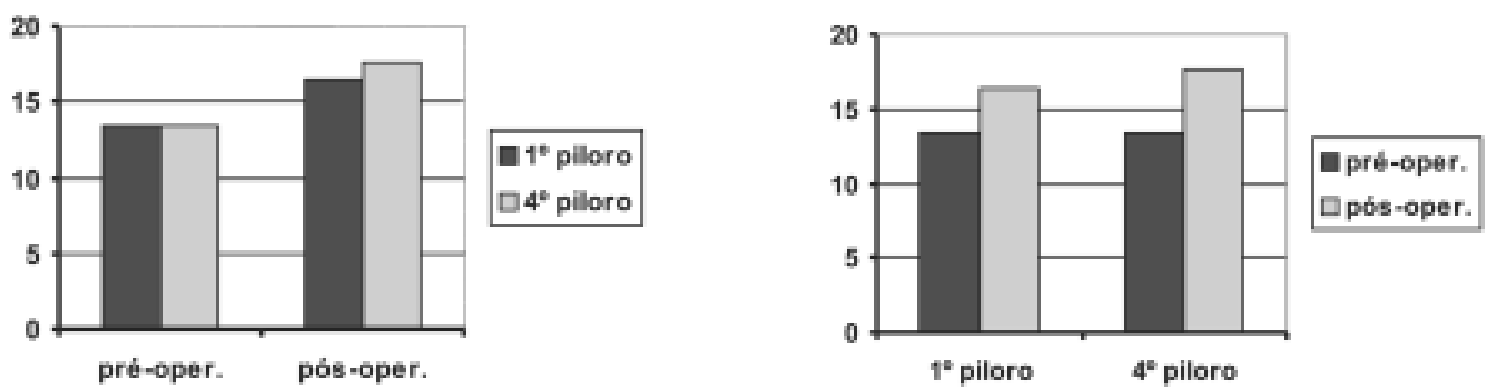

Gráficos 1 e 2 - Valores das médias das medidas das circunferências, em milímetros, das alças no pré e pós-operatório - Grupo 1. Evidenciando o aumento da medida quando comparados o pré e pós-operatório.

formalina a $10 \%$ e enviada para estudo anátomo-patológico.

No estudo anátomo-patológico das 40 peças de intestino de rato foram feitos cortes, um em cada prega identificada, no sentido perpendicular, em relação ao maior eixo do intestino.

\section{RESULTADOS}

No Grupo 1 (animais mortos no $15^{\circ}$ dia de pós-operatório), a medida da circunferência aferida, $1 \mathrm{~mm}$ a montante ao primeiro piloro, no pré-operatório, foi de $11 \mathrm{~mm}$ a $15 \mathrm{~mm}$, com média de $13,45 \mathrm{~mm}$ $(S=1,10)$ e no quarto piloro variou de $11 \mathrm{~mm}$ a $15 \mathrm{~mm}$, com média de $13,45 \mathrm{~mm}(\mathrm{~S}=1,28)$. No pós-operatório, a medida no primeiro piloro, variou de $12 \mathrm{~mm}$ a $21 \mathrm{~mm}$, com média de $16,45 \mathrm{~mm}(\mathrm{~S}=2,50),(\mathrm{p}<0,001)$ e no quarto piloro variou de $14 \mathrm{~mm}$ a $23 \mathrm{~mm}$, com média de 17,60mm $(S=2,74),(p<0,001)$ (Gráfico-1).

Nesse grupo, a variação da diferença na medida da circunferência (dilatação), entre o préoperatório e o pós-operatório, no primeiro piloro ficou entre $-1 \mathrm{~mm}$ e $9 \mathrm{~mm}$, com média de $3 \mathrm{~mm}(\mathrm{p}<0,006)$ e no quarto piloro variou de $0 \mathrm{~mm}$ a $9 \mathrm{~mm}$, com média de 4,15mm (p<0,006), Gráfico-2).
No Grupo 2 (animais mortos no $30^{\circ}$ dia de pós-operatório), a variação da medida da circunferência no pré-operatório foi de $12 \mathrm{~mm}$ a $17 \mathrm{~mm}$, com média de $14,10 \mathrm{~mm}(\mathrm{~S}=1,21)$ e no quarto piloro essa variação foi de $11 \mathrm{~mm}$ a $16 \mathrm{~mm}$, com média de $14,55 \mathrm{~mm}(\mathrm{~S}=1,39)$. No pós-operatório, a medida da circunferência no primeiro piloro ficou entre $15 \mathrm{~mm}$ e 48mm, com média de 21,60mm ( $\mathrm{S}=7,09)$, ( $\mathrm{p}<0,001)$ e no quarto piloro variou de $14 \mathrm{~mm}$ a $26 \mathrm{~mm}$, com média de 20,30mm ( $\mathrm{S}=3,21)$, ( $\mathrm{p}<0,001)$, Gráfico-3).

Nesse grupo, a variação da diferença na medida da circunferência entre o pré-operatório e o pós-operatório no primeiro piloro, ficou entre $1 \mathrm{~mm}$ e $33 \mathrm{~mm}$, com média de $7,5 \mathrm{~mm}(\mathrm{p}<0,006)$ e no quarto piloro ficou entre $0 \mathrm{~mm}$ e $12 \mathrm{~mm}$, com média de $5,75 \mathrm{~mm}(\mathrm{p}<0,006)$, (Gráfico-4).

Houve um óbito no quarto dia pós-operatório no Grupo 2, por peritonite, devido à fístula entérica no terceiro piloro artificial sem sinais de obstrução intestinal à necropsia. Este animal foi retirado do estudo e substituído por outro mantendo as mesmas condições do experimento.

Na necropsia, além da dilatação do íleo a montante dos neopiloros, encontramos a presença de bridas e/ou aderências em quase todos os ratos. Em
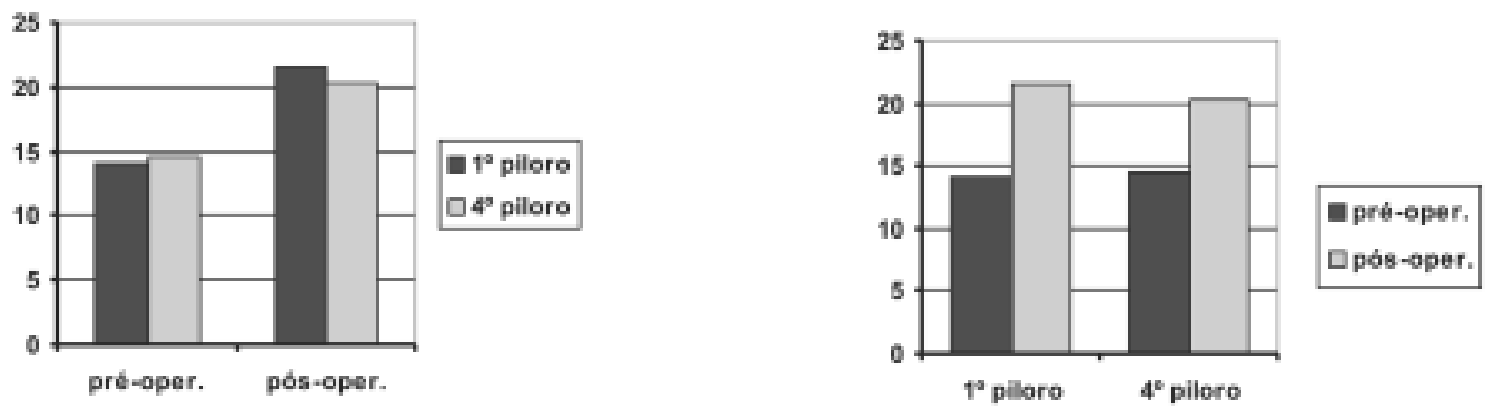

Gráficos 3 e 4 - Valores das médias das medidas das circunferências, em milímetros, das alças no pré e pós-operatório - Grupo 2. Evidenciando o aumento da medida quando comparados o pré e pós-operatório. 
ocorreu sub-oclusão intestinal, com presença de fístula enteroentérica. Em outro animal também encontramos fístula enteroentérica sem sinais de semioclusão intestinal. Ainda como complicação encontrada na necropsia, observou-se presença de um abscesso entre alças intestinais em um animal. Em nenhum caso, porém, essas complicações repercutiram a ponto de reduzir o peso ou a atividade dos animais (Figura-2).

Estiveram sob análise anátomo-patológica 140 pregas, sendo que destas, 59 mostraram-se proeminentes, 18 mostraram-se intermediárias e 29 mostraram-se rasantes. Não foram vistas 34 pregas. Em todos os casos, a parede intestinal apresentava-se ocupada por infiltrado inflamatório linfo-monoplasmocitário, compatível com o estágio de cicatrização e pela presença de granulomas do tipo corpo estranho. Em 62 pregas observou-se também infiltrado rico em polimorfonucleares, principalmente neutrófilos. 5 peças com 4 pregas cada foram perdidas durante o preparo para análise.

\section{DISCUSSÃO}

$\mathrm{Na}$ secção do intestino delgado, como em ressecções entéricas com anastomoses términoterminais, o complexo motor migratório ${ }^{4}$ é interrompido na área seccionada, mas novamente se origina no segmento distal por estímulo local, seguindo adiante sem nenhum prejuízo do conjunto peristáltico final, porém assume um ritmo próprio que é muito semelhante ao ditado pelo marcapasso localizado no duodeno ${ }^{5}$.
Tentativas de conter o trânsito aumentado com os reservatórios ou bolsas ileais usados em ileostomias e anastomoses íleo-anais, são válidas, apresentam bons resultados ${ }^{6,7}$, mas enfrentam, no entanto, a complexidade técnica da operação e o tempo cirúrgico prolongado ${ }^{8}$. Aqui ressaltamos o uso dos piloros naturais como, por exemplo, o piloro ileocecal $^{9,10}$. O uso de segmento em anisoperistalse não demonstra diminuição significante do trânsito, mas sim, em alguns estudos, aumento desse trânsito ${ }^{11}$. Em outros estudos há resultados satisfatórios ${ }^{12}$. Outras alternativas, como as alças de recirculação, não atingiram seu objetivo e, em alguns casos, houve piora do quadro clínico ${ }^{13}$.

Piloros artificiais nos parecem ser a melhor opção para reduzir o trânsito intestinal, por serem mais próximos da estrutura normal do tubo digestivo, reproduzindo mais a fisiologia dos processos digestivos, não interferindo com o complexo migratório motor, e regulando este trânsito intestinal.

Glassman foi o primeiro a descrever uma tentativa de reduzir o trânsito intestinal através de piloros artificiais em $1942^{14}$. Ele retirou $3 \mathrm{~cm}$ das camadas serosa e muscular longitudinal do intestino delgado, em ressecções intestinais amplas. Em seu trabalho a colonização do intestino remanescente era menor do que no grupo controle.

A retirada das camadas serosa e muscular longitudinal do intestino delgado com sutura da camada circular e aproximação das bordas cruentas foi descrita para tratamento de síndrome de "dumping" ou para minimizar os efeitos da síndrome do intestino curto ${ }^{2,15-17}$. A tática cirúrgica, entre esses

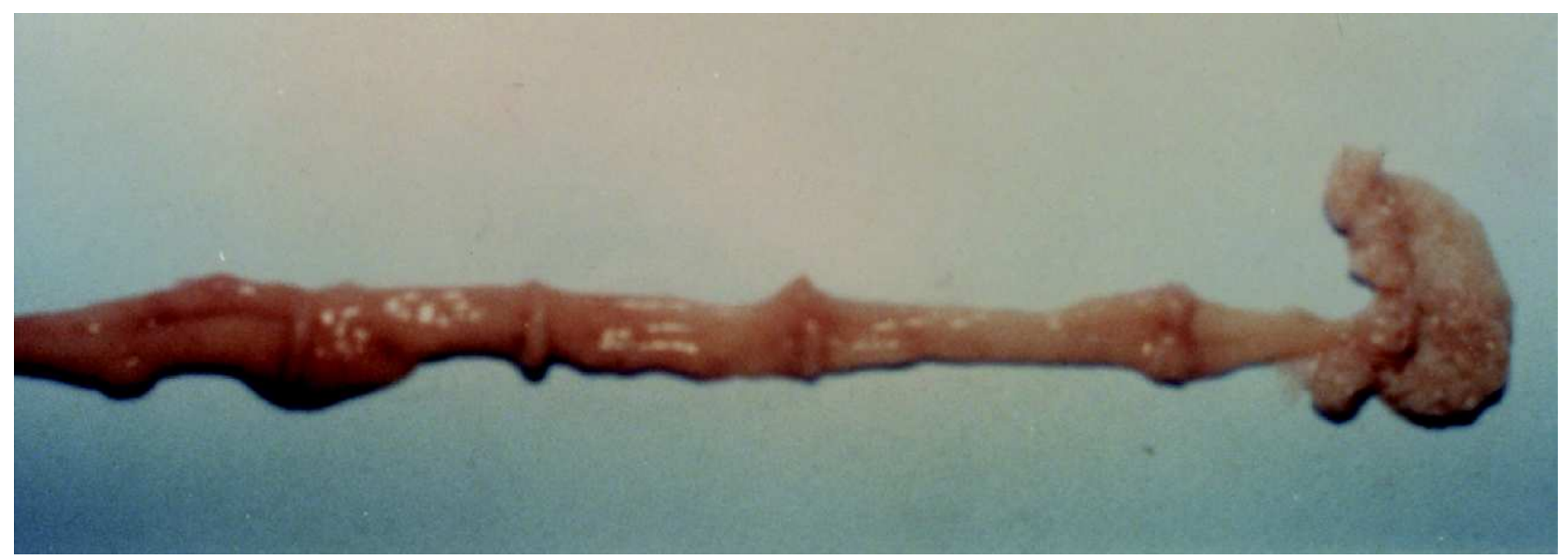

Figura 2 - Piloros bem definidos em intestino aberto visto pela face mucosa. 
autores, varia apenas na conservação ou não da camada muscular na face mesentérica, o que não mostra diferenças significantes.

Outra técnica para confecção de piloro artificial é realizada retirando-se além da serosa as duas camadas musculares do intestino delgado e invaginando a mucosa com sutura das bordas cruentas do intestino ${ }^{3,18}$.

Entre outras formas descritas, a secção de dois segmentos de serosa e musculatura longitudinal com agrupamento do segmento entre os dois fazendo um músculo dilatador maior é outra forma de se realizar piloros artificiais $^{19}$ e a secção e intussuscepção de segmento de intestino delgado, com eversão da porção distal do intestino seccionado ${ }^{20}$.

Estudos realizados com secção da mucosa mostram a manutenção do reflexo peristáltico com a simples secção da mucosa de forma circunferencial na alça intestinal ${ }^{21}$. Há evidencias que a barreira formada por piloros artificiais é mais mecânica que elétrica e não adianta, portanto, a secção do plexo nervoso entérico ${ }^{22}$

O uso de um piloro natural com sucesso para reduzir o trânsito intestinal, nos levou a pensar na possibilidade de criar um piloro ou um conjunto de piloros, uma vez que o piloro artificial não é tão eficaz quanto o natural. Propusemo-nos a fazer um estudo com técnica de fácil manuseio, sem solução de continuidade do intestino, somente seguindo a própria definição de DiDio sobre piloros ${ }^{23}$, tentando fazê-lo o mais próximo da normalidade.

Chrysospathis, em 1966, foi o primeiro a descrever a confecção de piloro artificial sem secção muscular, apenas pregueando a parede do estômago com pontos sero-musculares para prevenir síndrome de "dumping" 24 .

No nosso estudo, em que não seccionamos a musculatura, a dilatação do intestino delgado a montante dos quatro piloros reflete o sucesso em demonstrar que foram eficazes na diminuição do trânsito intestinal, evidenciando que não é necessária a secção de musculatura da parede do intestino para esse fim.

Não houve dificuldades na confecção dos piloros e nos nossos resultados a redução do trânsito é presente. Quando se estudam as outras propostas técnicas, observa-se que a que realizamos é de uma simplicidade maior.

\begin{abstract}
Background: To study, experimentally, the reduction of intestinal transit through artificial pylorus in terminal ileum of rats, without sectioning the enteric muscles. Methods: The study was carried out in two groups of 20 rats each. Four pylori were made in the terminal ileum of each animal with sero-muscular stitches circumferentially spread around the intestinal loop. Group 1 rats were killed after 15 days and those from group 2 after 30 days. The intestinal circumference was measured during surgery and postmortem.Results: Group 1 showed a mean $3 \mathrm{~mm}$ dilatation in the first pylorum and $4.15 \mathrm{~mm}$ in the fourth one. In group 2 the mean dilatation was $7.50 \mathrm{~mm}$ in the first pylorum and $5.75 \mathrm{~mm}$ in the fourth one. The presence of the well defined formation of the pylori were evident from anatomical studies. Conclusion: It is thus not necessary to remove or section the small intestine muscles nor section the intestinal neural plexus to promote intestinal dilatation and reduce intestinal transit with this method.
\end{abstract}

Key words: Ileum; Pylorus; Bowel Transit

\section{REFERÊNCIAS}

1. Becker JM - Distúrbios da Motilidade do Trato Grastrointestinal. Clin Cir Am Norte, 1994, 6, prefácio.

2. Schiller WR, DiDio LJA, Anderson MC - Production of artificial sphincters. Arch Surg, 1967, 95: 436-441.

3. Lázaro da Silva A - Tentativa de tratamento do "dumping” através de um "esfíncter" ileal. Rev Assoc Med Minas Gerais, 1974, 25(1): 32-33.
4. Szurszewski JH - A migrating electric complex of the canine small intestine. Am J Physiol, 1969, 217(6): 1757-1763.

5. Teleford GL, Walgenbach-Teleford S, Sarna SK Fisiopatologia da Motilidade do Intestino Delgado. Clin Cir Am Norte, 1993, 3: 1243-1250.

6. Keltikangas - Järvinen L, Järvinen HJ - Psychosocial adaptation to ileostomy: A study after construction of convention and continent stomies. Scand J Gastroent, 1987, 22: 202-206. 
7. Kock NG - Intra-abdominal "Reservoir" in patients with permanent ileostomy. Arch Surg, 1969, 99: 223-231.

8. Nicholls RJ - Restorative Proctocectomy with Various Types of Reservoir. Word J Surg, 1987, 11: 751-762.

9. Palma EC, Gonzales VH, Arias J. Ileostomia com esfíncter. Cir Urug, 1972, 42(4): 267-274.

10. Lázaro da Silva A - Ileostomia: alternativas técnicas. Rev Assoc Med Minas Gerais, 1978, 28: 28-29.

11. Sako K, Gerszi K, Marchetta FC - Nutritional effects of a short reversed jejunal segment. Arch Surg, 1964, 89: 1102-1105.

12. Shepard D - Antiperistaltic bowel segment in the treatment of the short bowel syndrome. Ann Surg, 1966, 163(6): 850-855.

13. Bianchi A - Intestinal loop lengthening - a technique for increasing small intestinal length. J Pediatr Surg, 1980, 15(2): 145-151.

14. Glassman JA - An artificial ileocecal valve. Surg Gyn. Obst, 1942, 74: 92-98.

15. Stachinni A - Contribuição para o estudo da anatomia funcional do intestino delgado do cão Tese de doutorado - Escola Paulista de Medicina USP, 1974.

16. Kapritchkoff E, Stachinni A, Cruz Filho M-Tratamento da síndrome de "dumping" - nova técnica cirúrgica "piloro artificial”. Arq Gastroent, 1977, 14(4): 224226.

17. Monteiro JA - Mecanismo valvular na síndrome do intestino curto, estudo experimental em cães. Tese de Doutorado, Universidade Federal do Rio de Janeiro 1986.
18. Ackroyd FW, Giles G, McDermott WVJ. Ileal mucosal valve to prevent reflux at the ileorectal anastomosis in the colon exclusion operation for hepatic encephalopathy. Surg Forum, 1969, 20: 356-359.

19. Rena CL, Lázaro da Silva A, Barra AA, et al. Seromiotomia dupla no intestino delgado: tentativa de criação de um piloro. Rev Col Bras Cir, 1996, 23(3): 143-147.

20. Careskey J, Weber TR, Grosfeld JL - Ileocecal valve replacement. Its effect on transit time, survival, and weight change after massive intestinal resection. Arch Surg, 1981, 116(5): 618-22.

21. Lin RCY, Schofield G - An investigation of the peristaltic reflex in relation to anatomical observation. Quarterly J Exp Physiol, 1958, 43(1): 26-37.

22. Zenilman ME, Parodi JE, Becker JM - Preservation and propagation of cyclic myoeletric activity after feeding in rat small intestine. Am J Physiol, 1992, 263: 248-253.

23. DiDio LJA - Piloros do Sistema Digestório. Rev Hosp NS Aparecida, 1950, 3(2): 55-104.

24. Chrysospathis P - Artificial pyloric canal. A modification of Billroth gastrectomy for prevention of dumping syndrome. Bull Soc Int Chir, 1966, 25(3): 257-265.

Endereço para correspondência:

Dr. Sergio Ibañez Nunes

R. Francisco Vaz de Magalhães 135/403

36033-340 - Juiz de Fora - MG

E-mail: sibanezn@terra.com.br 